\title{
TTR
}

Traduction, terminologie, re?daction

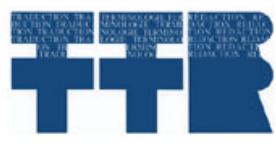

\section{Approprier la traduction}

\section{Gabriel Louis Moyal}

Volume 10, numéro 1, 1er semestre 1997

Langues, traduction et post-colonialisme

Languages, Translation and Post-Colonialism

URI : https://id.erudit.org/iderudit/037279ar

DOI : https://doi.org/10.7202/037279ar

Aller au sommaire du numéro

\section{Éditeur(s)}

Association canadienne de traductologie

ISSN

0835-8443 (imprimé)

1708-2188 (numérique)

Découvrir la revue

Citer cet article

Moyal, G. L. (1997). Approprier la traduction. TTR, 10(1), 19-52.

https://doi.org/10.7202/037279ar

\section{Résumé de l'article}

Approprier la traduction - L'étude de quelques articles publiés dans les pages du Constitutionnel et portant sur la traduction et la colonisation sont étudiés ici dans le but de montrer comment le pouvoir politique s'articule sur la traduction ou l'interprétation dans le discours de ce journal.

Tous les articles ont paru dans Le Constitutionnel entre mars et avril 1847, période qui correspond à la publication en feuilleton du dernier roman de Balzac, Le Cousin Pons. Ils portent sur des sujets très variés, couvrant une gamme qui comprend le statut légal de la traduction, les compétences qu'il faut avoir pour évaluer une traduction ou pour juger d'un savoir sur une nouvelle colonie, ou encore la bonne façon de raconter un massacre d'esclaves sur les côtes d'Afrique. Malgré cette disparité de sujets, quelques constantes s'avèrent sous-tendre l'approche que le journal adopte. On retrouve, à chaque fois, sous la discussion de ces sujets, une série de justifications politiques : celles du droit à la propriété et de l'expansion du capital, de la conquête et de l'exploitation des nouvelles colonies ainsi que du libéralisme économique. Le journal incorpore directement l'idéologie que représentent ces justifications dans son traitement de questions qui surgissent sur la traduction.

De façon plus générale, la stratégie dont use le quotidien pour établir son autorité et pour soutenir son idéologie consiste à ne fournir que sa traduction des événements ou des documents qu'il discute tout en décourageant ou en limitant l'accès aux faits ou aux originaux. Ainsi s'approprie-t-il la traduction : il fait passer sa version - laquelle implique toujours quelque naturalisation de la propriété - pour la seule convenable, la seule apte à transiger proprement avec la réalité.
Tous droits réservés ( ) TTR: traduction, terminologie, rédaction - Les auteurs, Ce document est protégé par la loi sur le droit d’auteur. L’utilisation des 1997 services d'Érudit (y compris la reproduction) est assujettie à sa politique d'utilisation que vous pouvez consulter en ligne.

https://apropos.erudit.org/fr/usagers/politique-dutilisation/ 


\section{Approprier la traduction}

\section{Gabriel Louis Moyal}

\section{Préliminaire : Présumer traduire}

J'ai reçu, Monsieur, avec bien de la reconnaissance et un vif intérêt, le bel ouvrage dont vous avez enrichi la littérature française et que vous avez eu l'attention de m'envoyer.

Si de puissans contrastes du caractère national exigent une grande diversité dans les formes propres à revêtir le sentiment et la pensée des poètes, cette difficulté augmente encore lorsqu'il est question de refondre en entier un ouvrage comme la Clarisse Harlowe de Samuel Richardson; de faire passer dans une autre langue un des chefs-d'ceuvre de la littérature anglaise, de reproduire, comme vous l'avez fait, Monsieur, avec succès, les beautés natives du style et la vigueur des premières conceptions.

[Lettre du $1^{\alpha}$ février 1847 de Frédéric-Guillaume, roi de Prusse, à Jules Janin, chroniqueur au Journal des Débats. $]^{1}$

Une recherche sur la publication en feuilleton du Cousin Pons de Balzac nous a amené à dépouiller à la fois Le Constitutionnel, journal où paraissait le feuilleton entre les 18 mars et 10 mai 1847 , et nombre d'autres quotidiens politiques français pour la même période. Il ne s'agissait pas initialement d'entreprendre une étude sur l'histoire de la traduction mais seulement de tenter de reconstituer, au sens le plus large

'Le Constitutionnel, 3 avril 1847, p. 3. Pour toutes les citations des journaux de cette période l'orthographe originale a été maintenue. Ainsi, par exemple, les mots se terminant en -ant ou -ent au singulier deviennent, respectivement, -ans et -ens au pluriel selon l'orthographe de cette période. 
du terme, un contexte ${ }^{2}$ qui permettrait de lire feuilleton et quotidiens comme deux versions d'une même histoire, deux reflets différents d'un monde, d'une société captés sur les mêmes pages au jour le jour et articulés l'un sur l'autre, avec leurs différences autant conventionnelles qu'idéologiques ${ }^{3}$.

Composés presque simultanément, feuilleton et journal s'évertuent, chacun à sa façon, de composer avec une réalité politique en pleine effervescence. A la veille des révolutions de 1848 , dans une monarchie de Juillet dont le déclin se voit précipité par des famines, par une crise économique sans précédent et une politique étrangère encore soumise à celles des alliés du Congrès de Vienne, on s'attendrait peu à voir l'un des plus importants journaux politiques de l'époque se soucier de la traduction. Or le fait même que Le Constitutionnel ait consacré à la traduction quelques articles pendant cette période s'avéra moins intéressant en soi que la façon dont ce journal en traitait ou, surtout, que les liens qu'on pouvait voir s'esquisser entre son discours sur la traduction et un discours idéologique plus explicitement axé sur les événements politiques du jour. Si ces quelques articles, portant de près ou de loin sur la traduction, pouvaient sembler, à première vue, de simples diversions, des contributions à la curiosité ou à la culture générale des abonnés du journal, ils devenaient, à les relire en contexte, représentatifs des stratégies discursives du journal. Introduits à titre de faits divers ${ }^{4}$, ils

\footnotetext{
${ }^{2}$ Voir, sur l'étendue à donner au contexte, Dominique Maingueneau, Le Contexte de l'cuvre littéraire : énonciation écrivain, société, Paris, Dunod, 1993.
}

${ }^{3}$ Pour l'histoire des journaux de cette période, voir Eugène Hatin, Bibliographie historique de la presse française, Paris, Anthropos, 1965, et Charles Ledré, La Presse à l'assaut de la monarchie : 1815-1848, Paris, Armand Colin, 1960.

${ }^{4}$ Les faits divers tiennent, sur un plan restreint, le même rôle que les romansfeuilletons : ils doivent distraire les lecteurs d'un contenu politique relativement lourd et piquer leur curiosité. Pour leur part, les romans-feuilletons n'obéissaient pas normalement à la ligne politique du joumal où ils paraissaient. Ils devaient principalement augmenter ou maintenir les chiffres d'abonnement du journal. Cela donnait souvent des résultats incongrus : c'est le cas de la publication du Cousin Pons dans Le Constitutionnel. Quand Balzac insérait, dans le cours de son roman, des attaques virulentes contre les libéraux qui avaient légué à la France la monarchie de Juillet, il accusait implicitement les rédacteurs du même joumal où paraissait le feuilleton. Voir, sur le rôle des feuilletons, Charles Ledré, op. cit. 
offraient, de par leur inévitable focalisation sur la langue et l'interprétation, une perspective autre sur le discours politique du quotidien. Intégrables d'abord à son idéologie, ces quelques articles en illustraient l'extension sur le plan du discours même.

Tel est le cas, par exemple, du fragment de lettre donné ici en exergue. La lettre, citée intégralement dans le journal, ne s'y accompagne d'aucune explication quant á la provenance du texte. Rien n'indique non plus d'autre justification pour cette publication qui, bien qu'adressée à un écrivain et journaliste bien connu, est d'abord une lettre personnelle. Mais le fait que Jules Janin était à la solde du Journal des Débats, lequel était en conflit ouvert à cette époque avec Le Constitutionnel, laisse présumer une certaine ironie quant aux raisons possibles derrière la diffusion de cette lettre ${ }^{5}$.

Mais encore, cette lettre, traitant de traduction, a-t-elle été confiée aux rédacteurs par le destinataire ou interceptée? A-t-elle été écrite en français par le roi lui-même ou traduite par l'un de ses secrétaires? Ou bien a-t-elle été traduite par un traducteur ou une traductrice anonyme, à la solde du journal - comme tous ceux et celles que Le Constitutionnel utilisait vraisemblablement, sans jamais les nommer, tous les jours, lorsqu'il reproduisait des extraits et des articles de journaux étrangers? Toutes ces carences, par ce qu'elles laissent présumer de sous-entendu, de conventionnel ou de normal dans les rapports entre le journal et ses lecteurs et lectrices, en disent long sur le statut de la traduction, comme de l'interprétation et du déchiffrage des textes.

Mis à part le contenu de la lettre, la façon dont le roi de Prusse parle de la traduction en français d'un best-seller anglais de l'époque n'est pas sans ressemblance avec l'attitude du journal. Même en faisant la part des politesses et des flatteries d'usage, on serait tenté de croire, à lire cette lettre, que Clarisse Harlowe serait tout bonnement devenu un roman français : Jules Janin en aurait, comme il dit, "enrichi la littérature

s On sait aussi que Frédéric-Guillaume ne donnait pas toujours aux journaux libéraux des raisons d'applaudir ses décisions politiques. Son refus persistant d'accorder une constitution à son peuple n'empêcha pas toutefois que l'ouverture du Parlement, avec son discours, soient publiés en détail dans plusieurs d'entre eux, y compris Le Constitutionnel, le 17 avril 1847. Voir plus loin le début de la section $\mathrm{V}$ du présent article. 
française", tant l'adaptation, la "refonte", la "reproduction" auraient été complètes. Il s'agit là, bien sûr, d'enrichir la «littérature" et non simplement la langue, comme l'auraient exprimé les clichés d'usage pour flatter une traduction. Mais aussi, ce fragment de lettre qui s'étend sur le procès même de la traduction - ce que Le Constitutionnel, normalement, ne fait pas - avance l'idée qu'une telle appropriation littéraire pourrait être la visée idéale d'une traduction. De cette entreprise, le roi indique les étapes, les traits essentiels. Il s'agit de ureproduire [...] les beautés natives du style et la vigueur des premières conceptions» ${ }^{6}$.

Mis à part les banalités propres au compliment, on peut se demander, devant l'absence de toute explication de la part du quotidien, ce qui peut se présumer des compétences du roi de Prusse en matière de traduction, ce qu'en cherche à transmettre Le Constitutionnel. Surtout que ce roi parle ici d'une traduction entre deux langues qui ne sont pas les siennes propres - si toutefois il aurait pu se dire, en matière de langues, propriétaire, ou possesseur - tout roi de Prusse qu'il est. Quelles qu'aient été, par cette reproduction d'une lettre privée, les intentions des rédacteurs du journal (ironiques, flatteuses, ou même désintéressées), il demeure que, par leur silence sur la traduction (en cette occasion précise surtout, mais aussi en toute autre), ils laissent sur ce sujet le roi parler pour eux. Ils le laissent parler de ce qui constítue une traduction idéale, des compétences qu'il faut avoir autant pour en faire une que pour évaluer celles des autres et, en plus, pour voir ses opinions publiées dans un grand journal parisien.

En l'absence de toute théorie explicite sur la traduction, ce qui fait donc loi s'avère être simplement la capacité d'en parler, de prendre la tribune pour désigner, pour une traduction idéale, une place dans la "littérature» de la langue d'arrivée. Ces appropriations qui, à bien considérer ce qui se trame dans les banalités flatteuses de la lettre, se voient démultipliées, correspondent, mis à part une certaine extravagance, aux attitudes cavalières qui se manifestent quotidiennement dans le discours du journal lorsque ce demier en vient à traiter, par exemple, des progrès de la colonisation de l'Algérie, ou de ceux de l'émancipation des esclaves en Martinique et en Guadeloupe.

${ }^{6}$ Le Constitutionnel, 3 avril 1847, p. 3. 
Non que Le Constitutionnel fasse montre d'une attitude cavalière par rapport à la propriété - loin de là. Pour autant qu'un journal libéral, anticlérical, puisse avoir une religion, Le Constitutionnel fait de la propriété la sienne. Mais il importe d'abord à ses rédacteurs de savoir à qui la propriété appartient et si ses titres se fondent sur une loi, un ordre, sur un système qu'il sait ou veut bien reconnaître pour légitime. Ainsi, par exemple, si la théorie de la traduction ne relève pas naturellement du domaine du roi de Prusse, il reste que, comme roi, tout ce qu'il a à dire est susceptible d'intéresser - même s'il ne s'agissait en cela que d'en faire ressortir le ridicule ${ }^{7}$. Par contre, lorsqu'il sera question des droits de propriété des indigènes algériens, les seules voix que ce journal considérera dignes d'entendre ou de publier seront celles du gouverneur-général et de ses officiers supérieurs, tous délégués par la France pour administrer et soumettre les territoires ${ }^{8}$. En cela, Le Constitutionnel - avec plusieurs autres journaux de l'époque - perpétue et contribue à affermir une hégémonie de pensée autour des principales questions politiques. Cette hégémonie assurera que toute mise en question éventuelle de l'arbitraire inhérent à la structure de ses raisonnements sur la propriété de territoires coloniaux ou sur la légitimité de la présence française dans ces régions, ne pourra se fonder sur aucune information fiable, sur aucune autorité reconnue. Ou, plus simplement, cette hégémonie rendra dissonant, au point de sembler être exprimé dans une langue étrangère, tout discours qui voudrait dénoncer telle disposition du territoire colonial.

Ainsi le droit de posséder, d'administrer des colonies, comme celui de définir ou de juger des bonnes traductions, se justifie-t-il, comme une compétence, par les faits accomplis. Mais aussi, il se consolide, se naturalise par l'occupation du terrain, par l'imposition d'une parole qui s'approprie, d'un seul et même geste, et le domaine et le droit d'en

\footnotetext{
${ }^{7}$ On pourrait comparer en ce sens les relations du roi de Bavière avec la chanteuse Lola Montès; relations dont les péripéties les plus absurdes défrayent, au long de la période étudiée, la chronique mondaine de nombre de journaux européens, et des plus sérieux - comme Le Constitutionnel.
}

${ }^{8}$ Voir dans la section III, aDresser, exproprier, colonisers, la note 22. 
discourir.. De même, on sait traduire ou évaluer les traductions parce qu'on occupe le champ, on parle à partir de cette place qui s'avère donner le droit d'en parler et même d'en juger, avec, en surplus, l'autorité : chaire, tribune ou rédaction d'un joumal dont la politique et la pratique s'accordent avec le jugement qu'on porte sur la traduction. Ce genre d'autorité, en quelque domaine que ce soit, devient gage d'expertise. On se l'approprie en prenant ou en se donnant la parole, ce que l'on peut faire le plus facilement lorsqu'on dirige ou possède le journal.

\section{Posséder}

À qui appartient une traduction? La question peut supposer un enjeu très vaste, amorcer, par exemple, un examen des rapports entre autorité et propriété. Elle ne provoque presque jamais de réponse simple mais renvoie toujours à au moins une double référence sinon une double appartenance. Elle entraîne, par rebondissement, la complication de schèmes reçus : ceux qui, de longtemps, veulent qu'on ne désigne qu'une identité simple, univoque, derrière un texte, un discours, une cuvre. Par là, la question de la propriété d'une traduction risque chaque fois d'engager encore, et dans une autre perspective, le débat sur l'auteur

${ }^{9}$ Cf. ce passage du Monolinguisme de l'autre qui projette cet effet sur le langage : «Car contrairement à ce qu'on est le plus souvent tentế de croire, le maître n'est rien. Et il n'a rien en propre. Parce que le maitre ne possède pas en propre, naturellement, ce qu'il appelle pourtant sa langue; parce que, quoi qu'il veuille ou fasse, il ne peut entretenir avec elle des rapports de propriété ou d'identité naturels, nationaux, congénjtaux, ontologiques; parce qu'il ne peut accréditer et dire cette appropriation qu'au cours d'un procès non naturel de constructions politico-phantasmatiques; parce que la langue n'est pas son bien naturel, par cela même il peut historiquement, à travers le viol d'une usurpation culturelle, c'est-à-dire toujours d'essence coloniale, feindre de se l'approprier pour l'imposer comme «la siennen. C'est là sa croyance, il veut la faire partager par la force ou par la ruse, il veut y faire croire, comme au miracle, par la rhétorique, l'école ou l'armée. Il lui suffit, par quelque moyen que ce soit, de se faire entendre, de faire marcher son aspeech act $n$, de creer des conditions pour cela, pour qu'il soit "heureux" ( $f$ elicitous* - ce qui veut dire, dans ce code, efficace, productif, efficient, générateur de l'événement escompté, mais parfois tout sauf aheureux) et le tour est jous, un premier tour en tout cas se sera joués; Jacques Derrida, Le Monolinguisme de l'autre, Paris, Galilee, 1996, pp. 45-46. 
comme sur l'origine des discours ou sur les enjeux des attributions ${ }^{10}$. Mais aussi, ce qui est sans doute plus menaçant, la question de la propriété d'une traduction risque, peut-être par ses prolongements, d'éveiller des doutes sur ce qui, de longue date, a constitué le droit à la propriété tout court. Par l'ambiguité des réponses qu'elle ne saurait éviter de donner à la question de la propriété, la traduction fait interroger certaines suppositions prises pour naturelles sur ce qui peut constituer un droit à la propriété. C'est peut-être pour éviter de telles mises en cause que l'on a eu tendance à poser la question indirectement, qu'on aura régulièrement cherché à y répondre par le biais d'autres questions.

En mars 1847 , un article de la chronique des tribunaux du Constitutionnel semble offrir une façon d'entamer la question avec, il semble, un minimum de risque et peut-être quelque avantage. Sous le titre «Une traduction est-elle une contrefaçon? $n$, l'article présente comme la réponse à cette question uintéressante et neuve" ${ }^{11}$, le jugement rendu par un tribunal français dans un procès intenté par les éditeurs d'un texte en français aux éditeurs français d'une traduction en espagnol :

Attendu, en droit, qu'il résulte des ar. 1 et 2 de la loi du 19 juillet 1793 , que l'auteur d'un ouvrage ou son cessionnaire en a la propriété exclusive, et qu'aux termes de l'art. 415 [sic] du code pénal, toute édition d'un ouvrage faite au mépris des lois ou règlemens relatifs à la propriété đes auteurs est une contrefaçon;

Que par leur généralité, des dispositions de loi s'appliquent à toute espèce de reproduction, lorsque cette reproduction est de nature à porter atteinte à la propriété d'autrui;

Attendu que la traduction d'un livre français en langue étrangère, reproduit nécessairement l'ouvrage original, puisque le traducteur en prend le titre et le sujet, les idées, les argumens et les phrases, tout en un mot, excepté la langue, et qu'il est évident que ce qui constitue un ouvrage, ce sont le sujet, les idées, l'ordre dans lequel elles sont

to Voir Michel Foucault, "Qu'est-ce qu'un auteur?», Bulletin de la Société française de philosophie, 1969, t. 63, $\mathrm{n}^{\circ} 3$; voir aussi Edwin Gentzler, "Foucault and De-structuring the Concept of Original in Contemporary Translation Theories, London \& New York, 1993, pp. 149-153; voir aussi Dominique Maingueneau, op. cit.

"Le Constitutionnel, 25 mars 1847, p. 4. Le texte en litige est la Défense du christianisme, ou Conférences sur la religion par M. Frayssinous. 
présentées et leurs développemens, et non l'idiome dans lequel il est écrit;

Que s'il est vrai que la traduction d'un livre en français n'est pas destinée à la même partie du public que l'ouvrage original, il n'en est pas moins certain qu'elle enlève à l'auteur, sans l'assentiment duquel elle a lieu, une classe de lecteurs à laquelle il aurait pu s'adresser, et qu'elle le prive des bénéfices sur lesquels il pourrait légitimement compter, soit en traduisant lui-même son ouvrage, soit en cédant moyennant rétribution, le droit de le traduire.

Que la traduction ainsi faite sans la pemission de l'auteur ou de son cessionnaire est donc une reproduction dommageable pour lui de l'ouvrage dont il a la propriété exclusive et présente par conséquent, lorsqu'elle est publiée ou débitée en France, tous les caractères constitutifs du délit de contrefaçon. ${ }^{12}$

Il est à noter que la traduction en question n'avait pas été autorisée et que la poursuite en contrefaçon est fondée sur cette absence d'autorisation. Néanmoins, les termes du jugement vont, on le voit, beaucoup plus loin que la simple question d'une autorisation. Tel qu'il se présente dans cet article, le jugement se donne pour tâche, il semble, de combler une lacune dans le code : l'absence d'une loi sur la propriété de la traduction. C'est ce sur quoi Le Constitutionnel attire l'attention en faisant ressortir la nouveauté de l'affaire. Le jugement, pour sa part, entend définir les liens qui relient l'original à la traduction (autorisée ou pas), et il le fait dans le cadre de la loi sur les contrefaçons. Pour cela, il représente la traduction comme une simple reproduction. De là cette insistance sur l'identité quasi totale proclamée entre original et traduction : utout en un mot excepté la langue». L'original ici se donne comme renfermant d'avance en soi toutes ses traductions et versions possibles et à venir. L'original est déjà (virtuellement) ses traductions, sauf dans un autre "idiome". Cette double exclusion de la langue de la traduction tend

${ }^{12}$ Le Constitutionnel, 25 mars $1847, \mathrm{p}$. 4. Le numéro 415 , donné pour celui de l'article pertinent du code pénal est sans doute l'effet d'une coquille. Dans tous les codes consultés de 1830 à 1968 l'article du code portant sur le droit d'auteur porte le numéro 425, auquel d'ailleurs l'article du Constitutionnel fait référence plus bas. Le texte de l'article 425 du code pénal se lit ainsi : aToute édition d'écrits, de composition musicale, de dessin, de peinture ou de tout autre production imprimée ou gravée en entier ou en partie, au mépris des lois et règlemens relatifs à la propriété des auteurs, est une contrefą̧on; et toute contrefaçon est un délit’, Les Sept codes des français, Toulouse, Rey, 1833. 
à neutraliser tout argument (présenté dans le procès ou que l'on chercherait éventuellement à présenter) visant à fonder l'originalité de la traduction sur la différence de langue, voire sur le travail des traducteurs.

Il n'y a sans doute pas lieu de supposer de préjugé contre la traduction en soi à partir d'un tel jugement. Plusieurs facteurs, circonstances et arguments mis en valeur lors du proces et qui ne sont plus disponibles à l'analyse auraient pu déterminer la rigueur dont la traduction fait l'objet ici. Reste toutefois ce jugement qui, pour trancher la question vraisemblablement trop ardue de la propriété d'une traduction, choisit d'enfler unilatéralement les droits de l'auteur de l'original (ou des éditeurs qui les achètent). La loi, telle qu'elle s'exprime ici, donne à l'auteur des droits qu'il n'aurait pu ou su anticiper : la traduction enlèverait à l'auteur wune classe de lecteurs à laquelle il aurait $p u$ s'adresser ${ }^{13}$. Elle fait de sa propriété un droit naturel (et infiniment extensible) offrant comme seule justification la contre-épreuve de l'atteinte portée à ce droit par une traduction non autorisée. La réaction semble disproportionnée à la provocation proférée par une traduction prise en faute d'autorisation. Ce seul délit qui fait parler la cour sur la traduction en général fait que, dans ce jugement, la traduction vient à paraître comme le résultat d'une opération mécanique. La traduction y est réduite, comme elle doit l'être pour tomber sous les termes de la loi sur la contrefaçon, au rang d'une areproductionn. Ainsi le travail de traduction se dessine-t-il ici comme le produit de quelque machine à reproduire, une copieuse qui projetterait simplement l'original en un autre idiome. Ayant ainsi situé la traduction, il devient plus facile, plus légitime, de dire que la traduction ne peut retenir que le droit d'acheter de l'auteur de l'original de quoi être, de quoi exister : «...soit en cédant moyennant rétribution, le droit de le traduire». Cette formulation ne fait, par son ambiguité, que reporter la question puisque la syntaxe de cette partie du jugement laisse indécis sur l'intention : qui rétribue qui et pour quoi, l'auteur pour la traduction, ou le traducteur pour le droit de traduire?

Comme la théorie de la traduction n'est pas régulièrement un des foyers d'intérêt des éditeurs du Constitutionnel de cette période, on peut se demander ce qui motive le choix de cette affaire juridique pour la chronique. D'autant plus que ce choix est marqué (comme il ne l'est pas

${ }^{13}$ Ibid., je souligne. 
normalement pour les affaires relevées dans la chronique des tribunaux) par l'ajout de la note annonçant l'inusité de la chose : «intéressante et neuve». L'intérêt et la nouveauté de cette affaire, chercherait-on à leur trouver quelque place dans la cohérence idéologique du quotidien, semble tenir à l'extension que ce jugement donne à une certaine forme de propriété plutôt qu'à une innocente curiosité de fait divers. Après tout, cette conception d'une propriété infiniment augmentable attribuée ici à l'auteur (et spécifiquement aux éditeurs auxquels il/elle l'aurait vendue) s'accorde bien avec l'évolution d'autres types de propriété, relativement nouveaux aussi, et qui s'avèrent également aptes à fasciner par leur capacité de s'étendre et de se découvrir des prolongements jusque-là inconnus ${ }^{14}$. Mais aussi, ce jugement qui statue sur la propriété de droit d'auteur fait abstraction du travail qu'on pourrait assumer avoir constitué ce droit. Ne sont mentionnés ni le travail de l'auteur et encore moins celui de la traductrice ou du traducteur. Le travail est passé sous silence en faveur de l'objet de la propriété en soi qui est, même si ni l'article ni le jugement ne le disent, le résultat d'un travail. Par là, ce jugement s'assimile à ce qui est une pratique courante à l'époque, non seulement du Constitutionnel mais de la plupart des quotidiens, soit de ne pas faire signer les articles publiés. Cette pratique s'étend d'ailleurs aux articles "reproduits" ou résumés de journaux étrangers, pour lesquels aucune attribution n'est normalement faite, ni pour l'auteur, ni pour la traductrice ou le traducteur. Ainsi, dans ce jugement, comme dans la pratique habituelle du journal, tout finit par se représenter comme si tel travail arrivait d'une pièce, sans effort ou labeur : possession de tous et d'aucun, reconnu comme propre de personne. Comme si, étrangement, cela n'était que de la "langue", de l'xidiome", que le joumal n'aurait eu qu'à cueillir tout fait dans quelque jardin du langage, comme si tel discours tenait de la nature et se prenait sans redevance, sans dette envers quiconque.

\section{Dresser, exproprier, coloniser}

Nous avons lu dernièrement, dans un journal d'Afrique, l'annonce d'un nouvel ouvrage où $\mathrm{M}$. le colonel Daumas, ancien capitaine instructeur

${ }^{14}$ La nature de la propriété intellectuelle est à elle seule une question très débattue en droit. Pour se faire une idée de l'étendue du débat, on pourra consulter la synthèse de Pierre Recht, Le Drout d'auteur : une nouvelle forme de propriété, histoire et théorie, Paris, Librairie générale de droit \& Gembloux, Éditions J. Duculot, 1969. Voir surtout pp. 48 et suivantes. 
de cavalerie, paraît s'être attaché surtout à nous faire connaître l'élève et l'éducation du cheval de guerre arabe. Cette publication ne peut manquer d'exciter à différents titres un très vif intérêt. Pittoresque d'un côté, instructive de l'autre, elle nous peindra probablement sous de vives couleurs les scènes poétiques de la tente; elle nous révélera sans doute aussi plus d'un secret utile pour la remonte et l'entretien de notre cavalerie d'Afrique. ${ }^{15}$

Ce qu'a de particulier ce passage sur l'élevage du cheval arabe c'est de venir, sans lien apparent, à la fin d'un autre compte rendu (c'en est le dernier paragraphe) d'un autre livre du lieutenant-colonel Daumas, «Directeur central des affaires arabes à Algen, plus récemment publié sous le titre de Le Sahara algérien. Les motifs de la publication de ce compte rendu laissent peu de doute : ce sera, selon l'auteur de l'article, un ouvrage d'une utilité rare pour la colonisation du territoire algérien. L'article, exceptionnellement, est signé : «le gérant : Ch. Marruau" [sic $]^{16}$.

Mais aussi ce paragraphe final du compte rendu, tout en anticipation, reprend, dans ce qu'il donne comme ses attentes, ce que, dans un domaine différent, le livre sur le Sahara algérien aurait déjà apporté : une expertise comme maîtrise, un savoir qui domine, qui sert et servira à dominer. Cette coda reprend même plusieurs des thèmes décoratifs du compte rendu : l'exotisme de la vie sous la tente des nomades, le mélange du pittoresque et de l'utile, etc. C'est donc aussi, d'une certaine façon, une traduction, une transposition, version équestre de ce qui, selon le gérant du Constitutionnel, assure la maîtrise présente et à venir d'une conquête. Implicitement, car il n'y a aucun besoin de le dire en toutes lettres, entre dresser les chevaux et dresser une colonie et ses indigènes à l'obéissance il n'y aurait qu'un pas, une mise au pas. Si ce paragraphe, qui fait aussi réclame, anticipe pour le livre à venir la reprise d'une vieille esthétique classique - le mélange de linstruction et du plaisir — le début du compte rendu en prône une nouvelle qui confond volontiers instruction et profit.

is Le Constitutionnel, 3 avril 1847, p. 3.

${ }^{16}$ Il s'agit vraisemblablement d'une coquille (Marruau au lieu de Merruau) : tous les historiens de la presse de l'époque nomment bien Charles Merruau (1807-1882) comme gérant du Constitutionnel. Poste qu'il garda depuis sa nomination par le Docteur Veron en 1844 jusqu'en 1849. Voir E. Hatin, op. cit. 
Mais la cohérence entre les deux parties du compte rendu est sans doute plus profonde, sans pour autant être intentionnelle, ou même consciente. Car ce qui ressort de cette juxtaposition c'est qu'en matière de colonies, comme en matière de chevaux, la possession se justifie par le savoir, le droit de dominer, de gouverner par la compétence, le savoir-faire. Cela s'établit dès le premier paragraphe de l'article qui, tout en décrivant les exceptionnelles compétences du colonel Daumas, se présume dispensé d'expliciter cette transition entre expertise et droit de propriété. Comme on peut d'un mêtne souffle parler et de la colonisation et du dressage des chevaux, on peut admirer les talents d'un haut fonctionnaire colonial sans vraisemblablement avoir à expliquer le fait même de la colonie. Ainsi, par le biais d'une évaluation de compétences, par une transposition du discours colonial en discours d'inspecteur général des écoles habilité avant tout à juger des compétences et de la science des autres, devient-il facile de rendre naturel, de traiter en évidence, l'appropriation d'un territoire habité. Cette stratégie de transposition s'entame, comme on peut le voir, dès le premier paragraphe du compte rendu :

Les bons ouvrages sur l'Algérie sont extrêmement rares, et cela se conçoit aisément : ni les études de cabinet, ni l'érudition commune, $\mathrm{ni}$ mêrne une intelligence supérieure, ne suffisent pour aborder un semblable sujet. Tant de conditions sont nécessaires pour s'éclairer soi-même avant de parvenir à éclairer les autres sur cette question tout exceptionnelle, qu'autant l'immense quantité d'ouvrages qu'elle a fait naître épouvante au premier coup d'oeil, autant attriste ensuite le petit nombre de ceux que la parfaite compétence de leur auteur recommande en effet à l'attention publique. Posséder le pays pour l'avoir traversé en tous sens, à diverses époques, en connaitre les habitans par stite de fréquens rapports avec eux et au moyen de l'entente parfaite de leur langue, s'être initié, s'il est possible, par l'exercice des fonctions publiques à leurs idées, à leurs lois, à leurs moeurs, dont un touriste ne peut atteindre que la superficie, voilà pour les conditions spéciales. Ajoutez un sentiment très haut de la mission que s'est donnée la France en Algérie, une grande indépendance, une grande sagacité d'esprit pour s'affranchir des préjugés aveugles de l'éducation sans toutefois s'égarer dans un monde nouveau, et enfin, les qualités essentielles du publiciste distingué, alors seulement vous aurez esquissé les grands traits auxquels 
on peut reconnaître le petit nombre d'écrivains téellement capables d'apporter des lumières nouvelles sur la question d'Afrique. ${ }^{17}$

Peu de sujets politiques obtiennent l'approbation quasi unanime des journaux politiques de cette période comme le fait celui de la colonisation de l'Algérie ${ }^{18}$. Toutefois, si autant de partis politiques sont en accord sur le principe de la conquête, ils le sont pour des raisons et des intentions différentes, chacun la justifiant selon telle composante de son idéologie et chacun cherchant, dans les rapports et écrits émanant des territoires conquis, les éléments qu'il lui faut pour justifier aussi sa position sur la politique intérieure de la France. L'Algérie devient par là une sorte de laboratoire idéologique, chaque parti voulant y trouver la confirmation de sa position et chacun la trouvant, presque infailliblement. Les contradictions nécessaires dans la représentation de ces différentes trouvailles marquent bien à quel point les journaux politiques, à ce stade de la colonisation, peuvent compter sur l'ignorance quasi totale, ou au moins la connivence, de leurs lecteurs ${ }^{19}$. De même, comme cette

\section{${ }^{17}$ Le Constitutionnel, 3 avril 1847, p. 3 , je souligne.}

${ }^{18}$ Pour la période étudiée (18 mars au 10 mai 1847), une douzaine de quotidiens recouvrant à peu près l'éventail des tendances politiques ont été dépouillés. $\mathrm{Si}$ quelques-uns critiquent telle nomination de fonctionnaire colonial ou telle décision de l'état-major quant à la stratégie à adopter pour soumettre les tribus, etc., aucun ne remet en question la présence de la France sur le territoire algérien ou son droit d'exploiter ce territoire.

${ }^{19}$ Avec cette connivence ne manque jamais de se marquer un sentiment de fierté, un regain du prestige national humilié depuis Waterloo. La conquête de l'Algérie doit racheter cette humiliation dont le ressentiment se marque régulièrement dans les éditoriaux. Ainsi cet article révélateur de L'Union monarchique : "Notre situation en Algérie a toujours été l'objet de la jalousie anglaise. Selon la politique de Londres, nous sommes des usurpateurs, [sic] qui avons envahi un pays, que nous aurions dû respecter. Toute idée de conquête nous est interdite. On veut bien vendre à Abd-el -Kader la poudre, les balles, les fusils, les canons et les boulets qui déciment nos soldats, mais on nous accuse de barbarie, lorsque, dans un engagement ou dans une razzia, nous tuons nos ennemis; et si par suite de la fatalité inexorable de la guerre, il faut imprimer la terreur à des populations barbares par un acte rigoureux mais nécessaire pour la sûreté ultérieure de nos troupes, alors c'est un chorus de malédictions : on nous dénonce au monde entier; on nous appelle sauvages. Mais ce qu'on nous reproche de faire en Afrique, on le fait sans remords dans les Indes. Nos lecteurs se rappellent la demière guerre des 
ignorance se double d'une curiosité aussi avide que la nouvelle colonie est vaste, les quotidiens ont chacun tout le loisir de la satisfaire à leur façon. De là pourront-ils aussi créer l'homogénéité dans l'opinion publique sur le principe même de la colonisation; homogénéité qui reste fondée sur l'ignorance ou, du moins, sur une connaissance transmise et filtree par les journaux et les partis politiques qu'ils représentent.

C'est en partie par cela que s'explique l'abondance d'ouvrages auxquels fait d'abord allusion le compte rendu. Ces projections idéologiques n'exigeant le plus souvent qu'une connaissance livresque (ce sont des aétudes de cabinetn), elles n'engagent pas nécessairement les auteurs à se rendre sur le terrain. M. Daumas, de par son rôle officiel et la longue durée de son séjour en Algérie, a déjà de grands avantages sur beaucoup de ces auteurs que l'article critique sans les nommer. Les louanges dont il est ici l'objet servent autant à le rapprocher du parti du Constitutionnel qu'à conférer à l'auteur du compte rendu son autorité. Il faut d'ailleurs noter que cette autorité ne se fonde pas explicitement sur une connaissance directe du territoire. Pour se permettre d'évacuer, en quelques phrases, tous ces autres écrits sur l'Algérie, l'auteur du compte rendu devrait normalement justifier son autorité. C'est ce qu'il ne fait pas et qu'il se gardera bien de faire ${ }^{20}$ même s'il accentue lui-même ce besoin : "Tant de conditions sont nécessaires pour s'éclairer soi-même avant de parvenir à éclairer les autres ${ }^{21}$. C'est sans doute pour esquiver la question de sa propre compétence - compétence dont il exprime ici lui-même l'essence : la nécessité d'une présence prolongée sur le terrain - que

sichks [sic] et l'envahissement du royaume de Lahore par l'Angleterre. Ce royaume n'existe plus que de nom. Ce n'est aujourd'hui qu'une province nouvelle ajoutée aux possessions de la compagnie anglaise”, LUnion monarchique, 4 avril 1847, p. 1.

${ }^{20}$ Rien dans les rubriques biographiques que nous avons pu consulter sur $\mathrm{M}$. Merruau n'indique sa présence possible en Algérie pendant ou avant cette période. Charles Merruau semble avoir passé sa vie d'abord dans l'enseignement ensuite dans l'administration. Il aurait été nommé, en 1840, secrétaire général de l'instruction publique. Un livre intitulé $L$ 'Égypte contemporaine (1858) est l'œuvre de son frère, Paul-François, lequel aurait aussi contribué à la rédaction du Constitutionnel.

${ }^{21}$ Le Constitutionnel, 3 avril 1847 , p. 3. 
l'auteur-gérant insiste tant sur l'incompétence manifeste dans la multitude d'ouvrages déjà parus. Mais surtout, c'est en mettant ainsi en valeur le savoir, les compétences du colonel, avec ce critère que le bon sens ne peut qu'approuver, que M. Merruau s'attribue implicitement - mais sans autre justification - une compétence égale — voire supérieure — sur l'Algérie. Il s'approprie, par ce savoir feint, la même autorité, les mêmes droits d'expertise qu'il attribue au colonel. Si les critères pour juger du savoir du colonel peuvent paraître raisonnables, ceux qui confèrent l'autorité de les établir à M. Merruau restent inconnus. S'ils existent quelque part ce ne peut être qu'en vertu de cette autorité que lui confêre sa place de gérant de journal; la même dont il use pour esquiver toute justification de la colonisation de l'Algérie. Cette logique tronquée donne suite à une série de raisonnements tout en implications, tout aussi boiteux.

Si pour les colonies - comme pour les chevaux - le savoir (ou le savoir-faire) confère l'autorité et justifie la possession, si ce savoir ne peut être validé que par une présence prolongée, - il faut *Posséder le pays pour l'avoir traversé en tous sens, à diverses époques" - ce que le texte ne dit pas c'est que ce long séjour n'a finalement été rendu possible que par une violente prise de possession ${ }^{22}$. Le savoir à lui seul ne saurait

${ }^{22}$ Le Constitutionnel, 3 avril 1847, p. 3, je souligne. Quelques jours avant la parution de ce compte rendu, le 30 mars 1847, Le Constitutionnel, comme plusieurs autres journaux politiques français, publiait une lettre circulaire du Maréchal Duc de L'Ysly, gouvemeur-général de l'Algérie, adressée aux généraux des trois divisions françaises en Algérie. On pouvait y lire plus ouvertement les rapports possibles entre le long séjour et la logique du conquérant victorieux sur la question de savoir ce qui finalement constitue un droit de propriété : *Je crois vous avoir dit plusieurs fois que ma doctrine politique vis-à-vis des arabes était, non pas de les refouler, mais de les méler à notre colonisation, non pas de les déposséder de toutes leurs terres pour les porter ailleurs, mais de les resserrer sur le territoire qu'ils possedent et dont ils jouissent depuis longtemps, lorsque ce territoire est disproportionné avec la population de la tribu.

Je considère la longue possession comme équivalente aux titres écrits et devant donner lieu aux mêmes ménagements, avec cette différence cependant que, lorsque les circonstances permettent de resserrer une tribu qui n'a d'autre titre qu'une longue jouissance, on peut se dispenser de lui donner des indemnités pour ce territoire qu'on lui prend; mais, même dans ce cas, il est convenable et politique de lui accorder quelques dédommagements pour l'espace qu'on lui enlève. Ces dédommagemens peuvent être un pont, un barrage pour les irrigations, une route, une mosquée; quelques secours en bois ou en fer, en ouvriers pour aider les 
conquérir de territoire autre qu'intellectuel. Et ici, ce savoir n'a pu exister qu'après la conquête et, par conséquent, ne saurait justifier cette dernière. Le jeu sur le sens intellectuel du verbe "posséder" ici, ne fait que véhiculer plus subtilement d'autres sens, plus proches de ceux qu'emporte la science équestre sur laquelle se conclura le compte rendu. L'inévitable aboutissement de cet argument qui cherche à justifier la prise de possession du territoire par la compétence, par la connaissance quasi parfaite du territoire n'est qu'une autre astuce discursive, analogue à celle du gérant-auteur qui ne justifie nulle part son droit de juger le livre du colonel ou ceux des autres. Il semblerait, contrairement à ce que laisserait croire le critère du long séjour ou celui de la connaissance de la langue des indigènes, qu'il suffit pour prétendre juger des connaissances des autres sur l'Algérie de le faire tout simplement, de montrer qu'on a compris, qu'on a su interpréter leur expertise, l'assujettir à nos critères universels du savoir et de la compétence.

En plus de prétendre savoir juger de la compétence élémentaire et générale du colonel ou de son livre, l'auteur du compte rendu se donne comme habilité - sans doute à partir de catégories empruntées dans le livre même - à déterminer et distinguer les différents degrés de compétences et leur place dans la hiérarchie d'un savoir - éventuellement complet - sur la colonie. En plus d'étendre l'esquive décrite plus haut autour du droit de juger que s'arroge, dans ce compte rendu, le gérant du Constitutionnel, cet accroissement immotivé de ses capacités laisse

indigènes à construire des villages, des habitations, des distributions d'arbres utiles pour planter les environs de leurs habitations, un fondouck reconnu nécessaire, enfin un objet quelconque d'utilité publique désiré par la tribu.

Ces compensations doivent d'autant moins être négligées qu'en même temps qu'elles satisferont à la politique, vis-à-vis de chaque tribu, elles concourront puissamment à la prospérité générale du pays", Le Constitutionnel, 30 mars 1847, p. 1. On retrouve encore cette logique du *long séjours comme titre de possession dans ce passage du Corsaire Satan : wLes députés qui font les grands raisonneurs sur l'Algérie sont d'ordinaire des honorables un peu présomptueux, et qui pensent, pour être allés quinze jours ou trois semaines par là-bas, avoir l'Afrique infuse. Tous leurs discours font un peu sourire les gens qui, par un long séjour dans notre colonie, possedent la clé des affaires et du caractère arabe», Le Corsaire Satan, 10 mai 1847. Ici encore, rien n'indique l'autorité de l'auteur - autorité qui est d'ailleurs reportée sur d'autres - néanmoins, rien ne saurait vraisemblablement ébranler son assurance quant à cette possession par le savoir de anotre colonien ou du caractère de ses habitants. 
quelque peu transparaître sur quels fondements, à partir de quels critères pourrait se légitimer l'évaluation d'un savoir sur un champ inconnu, l'examen de la possession intellectuelle d'un territoire inexplore jusque-là. Ainsi, vers la fin du paragraphe, le "voilà pour les conditions spéciales» qui suit la liste des efforts du colonel Daumas pour s'initier à la profondeur du territoire (le touriste n'ayant droit qu'à la usuperficien) en dit long sur ce que ce savoir présume comme transparence quant au savoir de l'autre. Ce n'est pas que les efforts du colonel soient inutiles ni même impertinents quant à un savoir possible sur l'Algérie, ou quant à une bonne volonté de comprendre un domaine étranger. Ce qui se marque dans cette clôture de liste est la présomption de pouvoir surmonter tous les obstacles concevables au savoir. Rien ne saurait dérouter l'entreprise du militaire devenu haut fonctionnaire, ou de toute autre personne pourvue de la même formation européenne, une fois payé le tribut de ces "conditions spéciales". Le savoir européen - parodiant ici le savoir cartésien - définit non seulement ses propres conditions mais aussi celles de l'essence (la profondeur) du savoir d'autrui. Tout savoir qu'il est, c'est là ce que semble présumer l'auteur-gérant, le savoir de l'autre, quel qu'il soit, ne saurait résister à l'exercice du savoir français. L'étendue de la liste qui précède le "voilà pour les conditions spéciales» donne la mesure relative de l'effort du colonel, marque son mérite. La transparence du territoire conquis à la méthodologie du conquérant - tout comme celle du travail du colonel au jugement du gérant - ne saurait faire de doute ${ }^{23}$.

${ }^{23}$ C'est ce qui semble échapper à M. Merruau, comme peut-être au colonel; l'application de ces grilles, de ces catégories d'un savoir européen ne sauraient livrer l'essentiel d'une matière qui leur est fondamentalement étrangère. Cela apparaît déjà, comme le trait d'une conscience vague, un peu plus loin dans ce compte rendu. Ainsi, dans la phrase suivante, laquelle tente de décrire la perspective inévitablement ambiguê de $M$. Daumas, apprend-on que celui-ci écrit avec «une grande sagacité d'esprit pour s'affranchir des préjugés aveugles de l'éducation sans toutefois s'égarer dans un monde nouveau’. Pour être sûr qu'il a trouvé la juste mesure, qu'il a su éviter ces deux écueils, les deux cécités que provoquent les préjugés de son origine et l'envoùtement du désert, il faudrait que ce texte puisse foumir un point d'appui, une garantie, un certificat de compétence quant à une sorte d'équité épistémologique possible. C'est ce qu'on est encore très loin de pouvoir assumer. Faute de garantie on pourra ici, a titre très strictement provisoire, avoir recours à un exemple, un échantillon d'enquête menée par le colonel que le texte de ce compte rendu nous fournit, comme si, tout naivement, il pouvait y avoir en cela quelque garantie d'intelligence, de sagacité, d'ouverture d'esprit, de bonne volonté. Quelques lignes plus loin, dans une sorte d'anecdote 
Les failles logiques évidentes dans ces deux arguments du compte rendu laissent sentir - si encore il fallait le démontrer l'impossibilité de justifier logiquement la prise de possession d'un pays habité. L'incompatibilité de la logique ou du savoir avec la violence physique et matérielle qu'entraîne inévitablement l'appropriation d'une colonie, fait que toute tentative de justification ne saurait révéler autre chose que lincompétence - en logique comme en savoir - de quiconque prétendrait avoir résolu cette aporie. Si cela n'entrave en rien la démarche du gérant signataire de l'article, il n'y a pas de raison pour autant de s'en laisser imposer par l'assurance du discours. Celle-ci ne tient qu'à la présomption et à la reconnaissance d'une communauté d'intérêts chez les abonnés du journal. Sans quoi on serait obligé de penser que les territoires algériens exerçaient quelque pouvoir d'envouttement sur l'esprit de l'auteur-gérant comme sur ses lecteurs pour expliquer l'inconscience à peine vraisemblable avec laquelle la logique, le sens critique des abonnés se seraient laissés escamoter. On peut alors se demander de quel avantage peut être, pour une colonie, une administration incapable de justifier de façon cohérente sa présence en territoire conquis ou trop confuse pour désigner simplement les intérêts qu'elle vient y chercher.

Néanmoins, même si on se laissait convaincre, par l'échafaudage des arguments qui autorisent dans ce texte l'élection du savoir comme critère ultime de la possession coloniale, cela n'empêcherait pas l'insuffisance de la justification de se faire quelque part sentir. Dans la mesure où ce compte rendu, pour se donner comme travail rationnel doit fatalement faire se confronter idéologie et raison, la justification ne pourra manquer de faire remarquer son absence. Il semblerait que, peut-être dans la foulée de ses recours apparents au sens commun, ou peut-être par un souci de forme ou de symétrie, M. Merruau se soit senti obligé de combler le vide. Mais la justification qu'il finit par donner ne relèvera pas du même registre que l'évaluation du livre. Elle ne fera pas appel à une logique cohérente ou tronquée - mais aux sentiments patriotiques, à l'émotion. C'est ainsi qu'apparaît cette mention du usentiment très haut de la mission

empruntée au texte, M. Merruau explique comment les chaouchs du colonel choisissaient eux-mêmes les personnages les plus exotiques du marché pour qu'il puisse les interroger à son aise. 
que s'est donnée la France en Algérie» ${ }^{24}$. Ce sentiment, le colonel Daumas l'aurait comme naturellement acquis. A en croire l'agencement du texte, il lui serait venu comme en surplus à son savoir-faire. Si M. Merruau daigne enfin nommer quelque justification de la présence française en Algérie, celle-ci ne relève plus des compétences, lesquelles entraînent l'investissement d'efforts, l'accomplissement d'un travail, de fonctions. Représentée comme sentiment d'identité, la justification s'associe (mais avec une naïveté qui tient aussi à ce qu'elle est déplacée dans le discours), aux attributs naturels du citoyen. Elle n'a à satisfaire aucune "condition spéciale” " ${ }^{25}$, elle est tout simplement là, comme un trait de caractère, de la personnalité du colonel, du même ordre que sa "grande sagacité ${ }^{26}$. Si donc la prise de possession de l'Algérie se justifie par l'acquisition d'un grand savoir et par des compétences exceptionnelles, c'est parce que ces derniers ne serviraient qu'à actualiser comme un instinct naturel, non pas, tout crûment, celui de la propriété, mais celui du devoir, de cette thaute mission" qui sert ici d'habit, de couverture au même instinct.

\section{Parler pour l'autre}

On pouvait lire, le même jour, dans le même numéro où $L e$ Constitutionnel publiait son compte rendu du livre du colonel Daumas et la lettre du roi de Prusse, les quelques paragraphes suivants sur un événement dramatique qui aurait eu lieu sur la côte africaine, à savoir le massacre de deux mille esclaves :

Sous le titre : Affreuses conséquences des croisières à la côte d'Afrique; le Courrier du Havre rapporte que deux mille nègres ont été massacrés par un marchand d'esclaves. Voici en quelles circonstances ce crime aurait été commis.

Au nombre des mesures adoptées pour la répression de la traite, dans les conférences qui ont eu lieu à Londres à l'époque oủ l'on a remplacé le droit de visite par une convention plus déplorable encore, le blocus des points de la côte où les négriers réunissent les esclaves de manière à pouvoir les embarquer au premier signal, figure en première ligne.

\footnotetext{
${ }^{24}$ Le Constitutionnel, 3 avril 1847, p. 3.

${ }^{25}$ Ibid.

${ }^{26} \mathrm{Ibid}$.
} 
Le commandant de l'escadre anglaise a été chargé, à ce qu'il paraît, de bloquer ainsi un ancien foyer de traite situé a l'embouchure d'une rivière appelée Gallinas. Or d'aprés le récit d'un officier britannique, cette mission aurait été remplie avec tant de succès, le blocus aurait été si hermétique, que le négrier, dans l'impuissance d'embarquer ses esclaves, aurait pris le parti de les tuer pour faire l'économie de leur nourriture. L'officier anglais a eu sans doute des informations inexactes. Il est difficile de croire qu'un Européen, même négrier, ait sacrifié de sang froid tant de victimes. D'ailleurs, cette cruelle avarice eût été mal entendue. La nourriture des nègres esclaves coûte bien peu à la côte d'Afrique. Deux mille de ces malheureux, si peu qu'ils coûtent, représentent cependant un capital assez considérable.

Les traitans savent bien que peu d'instans suffisent pour embarquer les noirs[sic]. Il paraît certain, en effet, que, malgré les croisières, la traite n'a rien perdu de son activité, et n'a pas cessé de procurer de très gros bénéfices.

Il n'est donc pas probable que le massacre de deux mille esclaves à Gallinas ait eu lieu par le fait d'un négrier européen. Mais il est très vraisemblable que ces malheureux auront été décapités par les ordres des chefs indigènes qui n'auront pas trouvé à les vendre. Ces scènes de carnage se renouvellent continuellement à la côte d'Afrique; il parất certain qu'elles deviennent plus fréquentes depuis que la vente des esclaves est plus difficile.

Voici ce qui arrive : les chefs africains amènent de l'intérieur des esclaves pour les vendre aux traitans etablis sur la côte. Ceux-ci refusent d'acheter cette marchandise vivante, ils se voient surveillés de trop près, s'ils n'attendent pas prochainement un bâtiment négrier. Alors les chefs africains tuent leurs prisonniers, par esprit de barbarie et par suite de l'impossibilité où ils sont de les nourrir. Puis, quand il se présente une occasion favorable, d'embarquer des esclaves, vite on fait une razzia aux environs; les forts s'emparent des faibles; le mari vend sa femme, le fils s'empare de son père par surprise, amis traînent leurs amis à la côte, et on entasse ces misérables créatures, pêle-mêle, dans la cale des négriers. En résumé, la traite ne diminue pas sensiblement, elle est seulement plus atroce. Tel est le résultat le plus clair du nouveau système de répression dont notre gouvernement s'est tant glorifié. ${ }^{27}$

Le trajet qu'aurait parcouru le reportage de cet événement semble particulièrement complexe même en tenant compte du fait que les moyens de transmission de l'information étaient relativement simples. En fait, à en suivre la trajectoire dans différents journaux, à comparer les différentes

${ }^{n 7}$ Le Constitutionnel, 3 avril 1847, p. 2. 
représentations dont cette information s'est avérée l'objet, on peut soupconner que ce sont les enjeux politiques qu'elle implique, les prises de position auxquelles elle a donné lieu, dans la presse, selon les intérêts politiques des divers journaux, qui ont assuré sa parution dans $L e$ Constitutionnel. Les deux dernières phrases de la version du Constitutionnel offrent de cela un échantillon éloquent. Par contre, plusieurs grands journaux politiques parisiens n'ont accordé aucune place à cet événement ${ }^{28}$. Le Constitutionnel, pour sa part, aurait repris la nouvelle du Courrier du Havre qui semble l'avoir lui-même reprise du Times de Londres. Le Times, qui fait préfacer l'article d'un petit commentaire éditorial, cite le Cork Constitution lequel, à son tour, dit que la nouvelle arrive d'Ascension, sans autre précision, et la fait dater du $1^{\text {er }}$ janvier $1847^{29}$. La première version française dont dit s'inspirer le Constitutionnel n'a pu être retrouvée, les numéros du Courrier du Havre pour les dates probables de sa parution n'étant plus disponibles ${ }^{30}$.

2! Certains journaux ne mentionnent ce drame que pour le démentir. Ainsi par exemple, le Journal des Débats, organe inavoué du gouvernement, sans avoir rapporté la nouvelle, publie le paragraphe suivant dans son numéro du 5-6 avril 1847: Le brick de guerre anglais le Cygnet, arrivé à Portsmouth pour y faire quelques réparations urgentes, a apporté en Angleterre des nouvelles de l'escadre de la côte occidentale d'Afrique. Elles démentent complètement une lettre publiée dans un journal irlandais et annonçant le massacre de 2000 nègres par des traitans qui n'avaient pu les embarquer. Malgré la vigilance des croisières, la traite se faisait encore avec une certaine activité. Pendant sa croisière, le Cygnet a arrêté dix-neuf navires ayant à bord 1760 esclaves. Sur ce nombre, quinze ont été condamnés comme négriers».

${ }^{29}$ The Times of London, 31 mars 1847 . Voir aussi, infra, la citation de cet article du Times.

31" La Bibliothèque Nationale (annexe de Versailles) ne dispose pas de tous les numéros du Courrier du Hovre pour cette période. Il n'a pas été possible de consulter les archives du Havre, seule autre source probable, avant la rédaction du présent article. Il s'avère néanmoins qu'un article dans le numéro du 4-5 avril du Courrier du Havre, lequel suit une lacune prolongée dans la collection de la BN-Versailles, cite l'article du Constitutionnel relatant ce massacre et signale ainsi l'influence marquante de l'article repris par le quotidien parisien : «La vérité se fait jour sur les résultats de notre croisière à la côte-d'Afrique, et Le Constitutionnel lui-même, qui n'envisage pas comme nous les questions relatives aux nègres, est forcé de reconnaître que leur sort a empiré depuis le blocus que nous avions mis à la suite de l'Angleterre, sur l'exportation de ces malheureux. Voici comment il 
$1847:$

Slave Trade - the following has just been received from a very intelligent naval officer at Ascension. The detail is one of the most appalling proofs of the calamities which the slave trade leads to, and we trust the efficiency of the naval blockade may put an end to its being continued in that part of Africa at least for some time to come. "Ascension, January 1, 1847 - we have just received news of a most horrible massacre on the coast. A slave depot called Gallinas, known to have 2000 slaves ready for shipping, was so closely blockaded by our cruisers that the slave owners finding it impossible to embark the slaves, and not wishing to incur the expense of feeding them, actually in cold blood beheaded the whole number, placing their heads on poles stuck in the beach, saying, if you will not allow us to make profit of prisoners we take in war, we will kill all". — Cork Constitution ${ }^{31}$

Quelles qu'aient été les raisons des péripéties, de la complication du trajet de cette nouvelle, il semble évident que, pour qu'elle ait été reprise par autant de journaux, il fallait qu'elle ait eu, malgré le retard considérable du reportage par rapport au moment des événements relatés, un effet considérable sur les rédacteurs et premiers lecteurs. Sauf qu'à considérer la façon dont, à chaque fois, l'information est encadrée, la portée des discours qui l'accompagnent, on ne peut s'empêcher de constater que la signification qui s'y rattache n'a plus tellement à voir avec le massacre de 2000 êtres humains. Le massacre en soi semble être devenu accessoire, s'être transformé en argument dans un débat renouvelé depuis peu sur l'esclavage, ou plutôt, dans le cas du gouvernement français, sur la question de l'émancipation des esclaves dans les colonies françaises.

Comme on pourrait s'en douter, par la mention que chaque version en fait, si ces événements semblent provoquer un renouveau du débat sur l'esclavage, cela tient maintenant à la décision unilatérale de l'Angleterre d'imposer un blocus des ports d'embarquement des bateaux des négriers sur la côte africaine. La suprématie de la marine britannique

s'exprime : [... suivent les deux demiers paragraphes de l'article du Constitutionnel cité plus haut]”, Le Courrier du Havre, 4-5 avril 1847, p. 1.

${ }^{31}$ The Times, 31 mars 1847 , p. 6. 
conférait à cette décision force de loi internationale et rendait toute opposition inutile. Même si, en d'autres circonstances, ils auraient tenu des discours plus favorables à l'émancipation, certains journaux de l'opposition libérale en France semblent s'être senti tenus de s'insurger contre cette ingérence britannique dans la politique coloniale française. Ils pouvaient vraisemblablement ainsi faire montre de leur nationalisme à coté d'autres journaux, plutôt conservateurs, qui allaient jusqu'à dénoncer comme étant à la solde de l'Angleterre les membres du gouvernement qui avaient proposé de renouveler le débat sur l'abolition de l'esclavage à la Chambre ou à l'Assemblée $e^{32}$.

C'est donc, inévitablement, dans le contexte de ces débats qu'il faut situer la publication de cet article et la forme très particulière qu'elle prend dans Le Constitutionnel. En même temps, on ne peut s'empêcher de reconnaître, dans la façon dont il reproduit l'article du Times (et cela, malgré l'apport qu'aurait pu avoir le Courrier du Havre), combien l'idéologie du Constitutionnel dépend d'un certain instinct de la propriété. Pour disculper les négriers européens, Le Constitutionnel retraduit les événements en leur imposant le test du bon sens commercial. Bien que, à la limite, on pourrait voir un contresens dans la volonté de proclamer l'innocence relative d'un négrier européen ( $\alpha$ il est difficile de croire qu'un Européen, même négrier, aurait pris le parti de les sacrifier pour faire l'économie de leur nourriture ${ }^{33}$ ), Le Constitutionnel semble pouvoir compter sur le sens commercial de ses lecteurs pour reconnaître la vérité de sa version lorsqu'il la fonde sur l'intérêt financier. Ainsi l'argument le

32 Voir, par exemple, l'article suivant du Corsaire Satan : «On a pu voir dans le grand Moniteur que l'ordre du jour de la chambre des pairs se trouve chargé d'une douzaine de pétitions réclamant l'abolition définitive de l'esclavage dans les colonies. M. le comte Beugnot, philanthrope émérite, est le rapporteur en titre de ces requêtes humanitaires. Personne ne pensait que ces [requêtes] pussent donner lieu à un incident parlementaire de grande importance, lorsque dans la séance du 13 mars dernier, $M$. le comte de Montalembert demanda à la chambre de fixer un jour spécial, qui serait consacré à la discussion de cet objet.

Plusieurs personnages éminents virent bien alors que la philanthropie anglaise, toujours si ardente à déprimer notre puissance coloniale, ne manquerait pas de souffler ses grandes théories sur le Luxembourg*, Le Corsaire Satan , 28 mars 1847, p. 1.

${ }^{33}$ Le Constitutionnel, 3 avril 1847, p. 2. 
plus convaincant n'est-il pas la race ou la conscience naturelle, mais l'instinct du bénéfice. C'est sur cet instinct, sur la reconnaissance de cet instinct entre initiés que compte Le Constitutionnel. Les abonnés du journal pourront désormais se faire confiance, l'instinct du bénéfice a, paradoxalement, des vertus civilisatrices - pour ne pas dire humanisantes :

[...] cette cruelle avarice eût été mal entenđue. La nourriture des nègres esclaves coûte bien peu à la côte d'Afrique. Deux mille de ces malheureux, si peu qu'ils coûtent, représentent cependant un capital assez considérable $[. . .]^{34}$

Les Africains qui, on doit le présumer ici, n'ont pas le même sens de la propriété, n'ont par conséquent pour partage que les instincts les plus bas pour guider leurs gestes, ordonner leur comportement : ales forts s'emparent des faibles; le mari vend sa femme, le fils s'empare de son père par surprise $[\ldots]{ }^{35}$.

L'étrange est que cette réattribution de culpabilité par voie d'une retraduction n'était pas nécessaire. Elle ne se justifie même pas dans une

${ }^{34}$ Le Constitutionnel, 3 avril 1847, p. 2.

${ }^{35}$ Le Constitutionnel, 3 avril 1847, p. 2. Ce genre de description des mours des Africains se retrouve, dans les mêmes termes ou presque, chez beaucoup d'auteurs européens du XIXe siècle - chez ceux surtout qui n'ont probablement jamais mis les pieds en Afrique. Elles sont généralement attribuées à des rapports de missionnaires lesquels, souvent, ne sont pas cités explicitement. C'est le cas, par exemple, de ce passage de Hegel qui explique ces mœurs par l'absence de concept divin : $\alpha$ [o]r chez les nègres les sentiments moraux sont tout $a े$ fait faibles ou, pour mieux dire, absolument inexistants. Les parents vendent leurs enfants et l'inverse se rencontre aussi, suivant qu'ils arrivent à se saisir les uns des autres. L'état général de l'esclavage fait disparaître tous les liens de respect que nous avons les uns pour les autres et les nègres n'ont pas l'idée de prétendre à ce que nous pouvons exiger réciproquement de nouss. Si Hegel reconnaît que ale seul lien essentiel que les nègres aient eu et ont encore avec les Européens, c'est celui de l'esclavagen, cela ne semble pas l'éclairer sur la relativité de ses connaissances sur eux : $\alpha$ En celui-ci [l'esclavage], les nègres ne voient rien qui ne leur convienne et les Anglais justement qui ont fait le plus pour l'abolition du commerce des esclaves et de l'esclavage sont trait's par eux-mêmes comme des ennemis", G. W. F. Hegel, Leçons sur la philosophie de l'histoire, traduction de J. Gibelin, Paris, J. Vrin, 1963, pp. 77 et 79 . 
opposition à la politique britannique ou dans la volonté d'imputer aux Anglais quelque hypocrisie, quelque fausse bonne conscience morale. Si les rédacteurs du Constitutionnel s'étaient donné la peine de lire l'article du Times, journal qui leur était normalement disponible (comme en témoignent nombre d'articles de ce dernier commentés presque quotidiennement dans les pages du Constitutionnel), ils se seraient aperçus que l'article anglais accusait déjà les Africains à la fois d'avoir eux-mêmes initié le commerce de l'esclavage et d'avoir commis le massacre en question. C'est ce qui se lit simplement dans la dernière phrase de l'article anglais. Il s'agit de "slave owners" et non de "traders", lesquels auraient acquis leurs marchandises par des guerres de tribus : "if you will not allow us to make profit of prisoners we take in war, we will kill all". Les rédacteurs du Times les avaient devancés dans cette entreprise de dégager les Européens de toute responsabilité quant au commerce des esclaves ${ }^{36}$.

Dans leur éditorial du même numéro, les rédacteurs du Constitutionnel semblaient d'abord faire montre d'une attitude bien plus éclairée quant à l'esclavage dans les colonies françaises. Ils défendaient le principe d'une loi unique et bien administrée tout en acceptant la nécessité d'une transition progressive vers une nouvelle économie dans ces colonies. Pourtant, cette attitude, pour avancée qu'elle paraisse, ne fait que remplacer une série de préjugés par une autre. À le lire de plus près, il s'avère que cet éditorial met, principalement sur les épaules de

${ }^{36}$ The Times, 31 mars 1847, p. 6. (je souligne, G. L. M.) Les histoires de l'esclavage rédigées au XIXe siècle cherchent, comme les rédacteurs du Times, à dégrever les Européens de responsabilité quant au commerce des esclaves en faisant remonter - du moins partiellement - ses origines aux mêmes sources que celles de l'esclavage en Europe dans l'Antiquité, c'est-à-dire à la capture de prisonniers de guerre. L'article $\alpha$ Esclavage* du Grand Dictionnaire Larousse du XIXe Siècle, vraisemblablement rédigé en 1871 - soit après que la France eut officiellement aboli l'esclavage même dans ses colonies - est représentatif de cette tendance. On peut y lire, malgré le ton moral qui y est de mise : "Ce fut la guerre qui fournit les esclaves, et depuis l'origine jusqu'à nos jours elle est derneurée la grande pourvoyeuse d'esclaves.

On comprend ainsi pourquoi on rencontre l'esclavage dans toutes les sociétés primitives. Il n'y a que là où toute organisation sociale fait absolument défaut et où le travail n'est pas devenu une fonction sociale imposée par la nécessité que l'esclavage n'existe pas. Ainsi le sauvage ne fait pas d'esclaves, mais il tue ses prisonniers de guerre et souvent même il les mange». 
propriétaires «créoles ou créolisés», à la fois le retard des réformes visant à l'émancipation des esclaves et les violences et injustices scandaleuses qui continuent d'être commises envers eux ${ }^{37}$.

${ }^{37}$ Voici le texte de cet éditorial du Constitutionnel du 3 avril 1847, (p. 1) : «Il est un point qui a vivement préoccupé la commission de la chambre des députés chargée, en 1845, de l'examen du projet de loi relatif au régime des esclaves dans les colonies : c'est la composition de la magistrature. Tous les hommes sensés comprennent que, dans l'établissement d'un régime transitoire entre l'esclavage et la liberté, le choix et la conduite des agens importent plus que les lois. Seuls ils peuvent amener les véritables progrès, et $\mathrm{si}$, par malheur, il y avait contradiction entre l'esprit de la magistrature et la législation nouvelle, on n'aurait créé dans les colonies que trouble, désordre et violence.

Nous ne sommes pas de ceux qui aiment à faire les philanthropes, à proclamer de grands principes stériles; mais nous souhaitons que sur tout le territoire français, la justice soit rendue avec impartialité, et qu'elle ne devienne pas une arme du fort contre le faible.

Deux choses étaient de nature à inquiéter vivement tous les hommes impartiaux. Les cours d'assises dans les colonies sont composées par quatre magistrats et trois assesseurs pris parmi les éligibles au conseil colonial, mais il faut cinq voix pour la condamnation, et ainsi, dans tous les crimes commis par les maîtres envers des esclaves, le concert des assesseurs peut et doit amener l'impunité. M. le ministre de la marine a lui-même déclaré à la chambre des députés, l'année dernière, qu'il s'était passế des faits scandaleux d'acquittement, et il a prié un honorable membre de ne pas en faire connaître le détail par ménagement pour lhonneur des tribunaux français.

On peut s'étonner après cela que le ministère n'ait pas songé à modifier sérieusement la loi réglant l'organisation des cours d'assises; mais il avait entre ses mains, sans le secours de la législature, le moyen de pallier une partie du mal. Il pouvait rendre la magistrature indépendante et digne de sa mission, c'est ce qu'il n'a pas fait.

D'après les déclarations même de l'administration auprès de la commission de la chambre des députés de 1845 , il y avait dans nos colonies soixante magistrats créles et un nombre un peu plus grand de métropolitains; mais parmi ceux-ci, beaucoup étaient mariés à des créoles ou possesseurs d'esclaves. Aussi la commission s'exprimait-elle ainsi : "Dans la métropole, des règles rigides garantissent l'impartialité des magistrats. Votre commission a cru qu'également des précautions devaient être prises aux colonies contre les faiblesses inhérentes à la nature humaine".

Et la commission ne s'est arrêtée que devant l'assurance formelle de $\mathrm{M}$. le ministre, que les choix futurs assureraient davantage l'impartialité des magistrats. Eh bien! sur les trente-huit mutations qui ont eu lieu dans les ressorts des cours royales de la Martinique et de la Guadeloupe, depuis le 11 décembre 
Si l'esclavage peut sembler inacceptable parce que inhumain, les préjugés

1845, jusqu'au 28 février 1847, 24 avancemens ont été donnés à des juges créoles ou créolisés, douze seulement à des magistrats métropolitains, et les deux seules disgrâces sont tombées sur des magistrats métropolitains.

Dans les deux cours royales, ta majorité appartient incontestablement aux réactionnaires. Si l'on entre plus avant dans le détail de l'organisation on verra que partout où le juge royal est un homme indépendant, le lieutenant de juge, c'est-à-dire le juge d'instruction, est animé d'un autre esprit, et que partout où le lieutenant de juge montre des dispositions à faire exécuter les lois, il est contrarié par la composition du tribunal ou du parquet. Quand on prend chaque tribunal dans son ensemble, on découvre avec douleur que la majorité appartient toujours à l'esprit réactionnaire.

Quelques magistrats créoles, il faut le dire, remplissent scrupuleusement leurs devoirs, mais par une étrange erreur de l'administration, ceux qui siégeaient à la Martinique et étaient originaires de la Guadeloupe, et réciproquement, ont été ramenés près de leur famille, et désormais subiront davantage les influences coloniales. Le nombre des juges de paix a été augmenté par la loi de 1845. On considérait ces magistrats cornme les véritables patrons des esclaves; ils ont pu l'être un moment, ils ne le seront plus à l'avenir. Sur les huit nouveaux choix, quatre sont tombés sur des créoles propriétaires d'esclaves.

Il faut le reconnaître la position des magistrats dans les colonies est très difficile. Un ou deux bons choix, tels que celui du procureur-général de la Martinique ne peuvent remédier au mal. Il faudrait que l'esprit de l'ensemble de la magistrature fut modifié et l'on a marché dans une voie contraire. Aux colonies, tout homme qui ne possède pas d'esclaves ou qui n'affiche pas les préjugés du possesseur d'esclaves, est considéré comme un individu de peu d'importance. Les sentimens d'insultante aristocratie dont la révolution de 89 a fait justice y règnent dans toute leur force avec leur intolérance. Le magistrat métropolitain qui débarque à la Martinique ou à la Guadeloupe, se trouve à l'instant même placé entre l'accomplissement de ses devoirs et les agrémens de la vie. Il ne jouira même d'aucune considération personnelle s'il ne renie pas les idées de sa patrie et les devoirs de son ministère; s'il ose penser ce qu'ici tout le monde pense, et croire que le droit du maître n'est pas illimité.

Il importe avant tout qu'un véritable esprit de justice et d'equité, que l'esprit du magistrat en un mot anime la justice coloniale, qu'il lutte contre les exagérations de l'esprit colonial. Ce spectacle ferait à lui seul plus que toutes les lois. Mais si, au contraire, tandis que la métropole promulgue des lois libérales, certains agens de la métropole sont animés d'instincts anti-libéraux, il y aura entre la loi écrite et la loi appliquée une déplorable contradiction, et au lieu de progrès modérés et prudens, du désordre et de l'anarchiem. 
raciaux eux demeurent incontestés ${ }^{38}$. C'est peut-être ce geste double qui reprend d'une main ce que l'autre donne qui remet en présence de ce qui est finalement la préoccupation principale des éditorialistes du Constitutionnel, c'est-à-dire la propriété privée. S'ils prônent l'émancipation en même temps qu'ils s'attaquent aux propriétaires créoles c'est que l'émancipation représente un avantage économique certain ${ }^{39}$. Le discours libéral sur lequel s'articule la protection de la propriété peut servir tout aussi facilement à accuser globalement les créoles de sauvagerie et d'antidémocratisme. C'est, sans doute, dans cette optique aussi qu'il faut lire leur revendication d'une justice qui usoit rendue avec impartialitén et qui ne devienne pas aune arme du fort contre le faible $w^{40}$. Telle revendication n'a pas pour fin, quelles que soient les prétentions exprimées, le bien-être des individus mais bien l'ordre qui permet la prospérité et la consolidation des droits des propriétaires.

Plus haut, le sens de la propriété, l'instinct du bénéfice devenaient les schibboleths, les talismans qui permettaient de substituer avec assurance une conjecture de propriétaire à un récit de témoin. C'étaient les signes par lesquels les rédacteurs du journal se faisaient forts de distinguer, sans hésitation, le comportement de l'Européen, de celui de I'Africain. Ce même instinct sert maintenant à distinguer l'administrateur ou le magistrat métropolitain du maître "créole ou créolisé" dont les instincts s'avèrent, lorsqu'il s'agit de reconnaître l'avantage économique de l'émancipation, mélangés, obscurcis. Dans la nouvelle optique du journal, c'est ce mélange qui maintenant menace le progrès, ou l'ordre propice à l'accroissement des profits.

\section{Traduire la propriété}

Les États-généraux viennent de s'ouvrir à Berlin, comme s'ouvrirent les États-généraux de France en 1789. Cest aussi un roi aimé de la nation, qui, entraîné par le mouvement libéral de son siècle, prend l'initiative de cette convocation. [...]

${ }^{38}$ Voir aussi, sur le discours français sur la race, Alain Ruscio, op. cit.

${ }^{39}$ Voir, entre autres, l'article cité du Larousse XLXe siècle sur les avantages économiques de l'émancipation.

${ }^{40}$ Le Constitutionnel, 3 avril 1847, p. 1. 
Le roi déclare 20 fois dans son discours, et avec la plus grande énergie de langage, qu'xaucune puissance sur la terre ne l'amènera jamais à changer les rapports naturels entre lui et son peuple en rapports conventionnels et constitutionnels garantis par des chartes et scellés par des sermens. Jamais il ne permettra qu'une feuille écrite vienne s'interposer entre Dieu et la Prusse, pour gouverner ce pays par ses paragraphes. ${ }^{41}$

Ce qui se présente comme traduction, laisse du moins présumer l'existence d'un texte de départ, d'un original. I1 serait sans doute légitime d'affirmer que les façons qu'a Le Constitutionnel de traiter les traductions auxquelles il a recours pour informer ses lecteurs ou avancer ses opinions politiques ne lui sont pas uniques. La traduction, on l'a vu, atteint à peine à cette époque au statut de la légalité. Elle est loin de hanter la conscience des lecteurs des grands journaux bourgeois. Assumer les textes traduits de langues étrangères comme si leur sens était transparent, comme si leurs représentations en français n'avaient posé et ne sauraient poser, une fois qu'ils sont traduits, de problème d'interprétation, de doute quant aux intentions essentielles de l'original est finalement une attitude que $L e$ Constitutionnel n'est pas le seul journal politique de l'époque à adopter.

Mais cette transparence assumée ne saurait laisser croire à une naïveté généralisée, à une confiance globalement et volontairement accordée aux rédacteurs et traducteurs des journaux. Pour se constituer et s'instaurer comme mode d'opération normal par rapport aux événements étrangers, accessibles initialement qu'en langue étrangère, il a fallu non seulement le besoin de facilité, d'accès aux renseignements, mais aussi une certaine complicité, une communauté de croyance en la suprématie de la langue nationale - comme de la nation même. $\mathrm{Si}$, dans les pratiques des journaux, l'original semble d'abord avoir si peu d'importance c'est aussi que toute perspective sur le monde autre que celle que fondent et que font partager les grands journaux est, par définition, de peu d'intérêt. Cet ethnocentrisme n'est ni le résultat d'une génération spontanée, ni

${ }^{41}$ Le Constitutionnel, 17 avril 1847, p. 1. Tout en prétendant vouloir s'abstenir de discuter les athéories* du monarque, la rédaction du journal ajoute néanmoins à ce reportage de l'ouverture du pariement de Berlin quelques commentaires. Elle fait remarquer, par exemple, que malgré le refus d'adopter une constitution écrite, le monarque accorde néanmoins à son peuple les droits de liberté de religion, de liberté de la presse, etc., les seules importantes selon l'auteur de l'article. 
simplement l'effet d'une indifférence massive d'une culture par rapport à une autre. Il s'articule sur une compréhension du monde comme lieu de conflit, comme étendue de territoires riches que les états européens les plus forts n'ont qu'à se départager afin de pouvoir les exploiter en toute quiétude $^{42}$. Dans une telle optique, seul importe ce qui a trait aux bénéfices, à la protection des investissements : seuls enjeux pertinents des relations avec d'autres cultures - qu'elles soient perçues comme concurrentes ou seulement comme victimes éventuelles. Les subtilités et raffinements d'une langue étrangère qu'une traduction négligente pourrait avoir escamotés risquent peu d'inquiéter les lecteurs.

On se croirait donc loin ici des préoccupations toutes littéraires qu'exprimait le roi de Prusse dans son compliment à Jules Janin. «Les beautés natives du style et la vigueur des premières conceptions ${ }^{43}$ ont peu à voir avec la circulation de valeurs marchandes, question qui alimente finalement le plus les polémiques politiques pour lesquelles se passionnent les lecteurs de journaux bourgeois à la veille de la révolution de 1848. Les considérations de valeurs poétiques ne semblent pas avoir souvent décidé de leur abonnement aux grands quotidiens.

Pourtant, le roi de Prusse et les éditeurs de ces quotidiens semblent avoir compris les mêmes choses en ce qui a trait aux liens éventuels entre l'original, la traduction et le pouvoir. Là où il s'agit de protéger son autorité, il est sage de ne pas offrir trop directement aux regards de tous et chacun l'original - qu'il soit texte ou tout autre symbole tangible - sur lequel telle autorité se dit fondée. Ainsi, dans l'exergue cité en début de section, le roi veut bien accorder un bon nombre des libertés civiles que lui réclament ses sujets. Mais il ne leur accordera pas de constitution - objet auquel il se réfêre, sans doute avec uénergie" et comme par dérision, sous sa forme matérielle la plus simple de wfeuille écrite", de "paragraphes", etc. ${ }^{44} \dot{A}$ sa façon toute particulière de l'exprimer, on comprend aisément la réticence du roi de Prusse : si chacun peut lire le document qui lui garantit ses droits de citoyens, chacun est en mesure

\footnotetext{
${ }^{42}$ Voir, à titre d'exemple, la citation de l'Union monarchique, note 19.

${ }^{43}$ Le Constitutionnel, 3 avril 1847, p. 3 , et cité au début de cette étude.

${ }^{44}$ Le Constitutionnel, 17 avril 1847, p. 1.
} 
alors de les interpréter à sa façon. Une fois la «feuillew substituée à ce souverain qui donne selon son plaisir ou sa bonne volonté, chacun se croira pouvoir revendiquer un droit, l'accomplissement d'une promesse, d'un contrat qui lierait le roi. Cette comparaison entre l'assemblée convoquée par Louis XVI et celle des états prussiens qui semble comme s'imposer au journaliste du Constitutionnel n'est pas déplacée : elle en dit aussi long sur l'intuition politique du roi que sur celle de la rédaction du journal :

Ces rapprochemens [entre la France de 1789 et la Prusse de 1847], quoiqu'ils soient plus apparens que réels, et qu'il y ait de très profondes différences entre les deux nations, les deux époques et les deux souverains, n'en paraissent pas moins avoir produit une très vive impression sur l'esprit du roi de Prusse. Le discours qu'il a prononcé à l'ouverture des Etats, semble inspiré tout entier par l'aversion des principes libéraux français, par le terrible souvenir de la Constituante et de Louis XVI. ${ }^{45}$

L'histoire de Louis XVI, on le voit, dicte à Frédéric-Guillaume cette sage économie de "feuille écriten. Son autorité qui reste, malgré quelques concessions, relativement intacte, il ne croit pas devoir y imposer de limites. En tant que monarchie héréditaire cette autorité dépend uniquement des forces militaires, policières ou autres qu'elle peut assembler pour se maintenir au pouvoir. Il ne tient encore qu'au roi d'accorder le texte, la constitution qui en définirait l'étendue.

Par contre, sur quelle autorité peuvent s'appuyer M. Merruau ou les autres rédacteurs de quotidiens bourgeois comme Le Constitutionnel lorsqu'ils évaluent tel savoir sur l'Algérie ou qu'ils pensent devoir amender l'explication d'un massacre dont ils n'ont pas été les témoins? Si des garanties constitutionnelles, telle celle de la liberté de la presse, leur octroient une certaine latitude quant à ce qu'ils peuvent publier, les compétences sur lesquelles devraient logiquement se fonder leur discours sont d'une autre sorte. Ils ne peuvent baser leurs prises de position sur un argument d'autorité sacrée, de révélation ou d'élection ancestrale puisqu'ils s'évertuent justement à discréditer de tels arguments lorsque leurs

${ }^{45} \mathrm{Ibid}$. 
opposants monarchistes ou conservateurs les avancent ${ }^{46} . \AA$ la différence de celui du roi, l'ordre que ces rédacteurs politiques cherchent à instaurer ne saurait faire appel à des valeurs spirituelles, à des traditions ou doctrines religieuses comme celle du droit divin. Ce qui les inspire et qu'ils ont à protéger - la propriété — n'a pas de justification inhérente. Si certains documents peuvent y garantir le droit, encore leur faut-il savoir en régulariser l'interprétation, en contenir et en diriger le sens à l'avantage de la classe dont ils protègent les intérêts. Aussi leur faut-il dissimuler les fondements de ces droits à la propriété qui trop souvent s'avèrent tout aussi arbitraires que ceux dont se réclament les princes héréditaires.

C'est pourquoi il importe à ces journalistes bourgeois et libéraux de s'approprier la traduction, de se réserver exclusivement le droit d'interpréter, d'évaluer non pas en s'accumulant des compétences herméneutiques mais en occultant l'original - qu'il soit texte, témoignage ou même expérience d'un «long séjour". En cela ils n'inventent pas : il leur suffit de reprendre les stratégies de l'ordre monarchique qu'ils veulent supplanter. Si ce dernier devait fonder son autorité sur un au-delà inaccessible, il suffit à ces nouveaux maîtres de contrôler l'accès à ce qu'ils disent traduire ou expliquer, de n'en donner que la version qui leur paraît la plus profitable tout en la faisant passer pour savoir ou science.

Dans son introduction à Translation/History/Culture, André Lefevere décrivait ainsi l'enchevêtrement de rapports de forces et de pouvoirs dans l'histoire de la traduction :

Translation has to do with authority and legitimacy and, ultimately, with power, which is precisely why it has been and continues to be the subject of so many acrimonious debates. Translation is not just a "window opened on another world", or some such pious platitude. Rather, translation is a channel opened, often not without a certain

${ }^{46} \mathrm{Par}$ exemple, dans cet article même, Le Constitutionnel cite le discours du roi qui prétend régner ad'après la loi de Dieu et du pays, et d'après sa propre détermination [...]" pour ensuite s'en distancer : *on comprend que nous ne discutions pas ici ces théories qui sont si fort en contradiction avec nos idées en matière de droit naturel, de droit politique et de libertén, Le Constitutionnel, 17 avril 1847, p. 1. 
reluctance, through which foreign influences can penetrate the native culture, challenge it and even contribute to subverting it. ${ }^{47}$

Ce que Lefevere dit ici des rapports éventuels entre des cultures différentes semble s'appliquer tout aussi bien à l'intérieur d'une même société. Non seulement lorsque celle-ci se met à s'inféoder d'autres peuples, à coloniser de nouveaux territoires, mais aussi entre les classes d'une même société, entre ses citoyens et ses gouvernants, ses possédants et ses exploités. Dans ses rapports à d'autres cultures - ce qu'une approche de la traduction conçue selon les lignes qu'esquissait Lefevere serait particulièrement apte à analyser - se révèlent en même temps les valeurs et principes, l'éthique et la politique qui organisent aussi les rapports internes de cette société.

\section{Université McMaster}

RÉSUMÉ : Approprier la traduction - L'étude de quelques articles publiés dans les pages du Constitutionnel et portant sur la traduction et la colonisation sont étudiés ici dans le but de montrer comment le pouvoir politique s'articule sur la traduction ou l'interprétation dans le discours de ce joumal.

Tous les articles ont paru dans Le Constitutionnel entre mars et avril 1847, période qui correspond à la publication en feuilleton du demier roman de Balzac, Le Cousin Pons. Ils portent sur des sujets très variés, couvrant une gamme qui comprend le statut légal de la traduction, les compétences qu'il faut avoir pour évaluer une traduction ou pour juger d'un savoir sur une nouvelle colonie, ou encore la bonne façon de raconter un massacre d'esclaves sur les côtes d'Afrique. Malgré cette disparité de sujets, quelques constantes s'avèrent sous-tendre l'approche que le journal adopte. On retrouve, à chaque fois, sous la discussion de ces sujets, une série de justifications politiques : celles du droit à la propriété et de l'expansion du capital, de la conquête et de l'exploitation des nouvelles colonies ainsi que du libéralisme économique. Le journal incorpore directement l'idéologie que représentent ces justifications dans son traitement de questions qui surgissent sur la traduction.

${ }^{47}$ André Lefevere, Translation/History/Culture : A Sourcebook, London \& New York, Routledge, 1992, p. 2. 
De façon plus générale, la stratégie dont use le quotidien pour établir son autorité et pour soutenir son idéologie consiste à ne fournir que sa traduction des événements ou des documents qu'il discute tout en décourageant ou en limitant l'accès aux faits ou aux originaux. Ainsi s'approprie-t-il la traduction : il fait passer sa version - laquelle implique toujours quelque naturalisation de la propriété - pour la seule convenable, la seule apte à transiger proprement avec la réalité.

ABSTRACT : Appropriating Translation - Through a study of selected articles bearing on translation and colonization in a French newspaper (Le Constitutionnel), this paper attempts to show how political power and interpretation or translation are articulated in journalistic discourse.

The articles studied all appeared in the paper in a period of several weeks in March-April 1847, a period which coincides with the publication in serial form of H. de Balzac's last novel (Le Cousin Pons). They cover subjects as varied as the legal status of translation, the competence to evaluate translation and knowledge about newly annexed territories, the proper reporting of a slave massacre as well as the possible liberalization of the Prussian monarchy. A number of constants are found to underlie all these issues as the newspaper raises them : the political justification of property, the rationalization of capital's expansion, the exploitation of colonies, the defense of economic liberalism. All of these are found to be homologous with the discursive strategies the newspaper employs to contend with the few instances of translation it deals with.

The primary stratagem the daily uses to establish its authority and to justify its ideology - consists in presenting only its own translation of the documents and events while restricting or discouraging access to originals. In so doing, the newspaper aappropriates» translation : it makes its version (which, at some level, implies a naturalization of property) appear as the only proper one.

Gabriel Louis Moyal : Département de français, Université McMaster, 1280 Main Street West, Hamilton, Ontario, L8S 4M2, courriel: moyalg@mcmail.cis.memaster.ca 